\title{
Attention should be paid to D-Dimer test in the management of COVID-19
}

\author{
Peng Zhou ${ }^{1}$, Qin $\mathrm{Li}^{1}$, Chao Yang ${ }^{1}$, Yiqing $\mathrm{Li}^{1}$, and Shi Chen ${ }^{1}$ \\ ${ }^{1}$ Affiliation not available
}

July 29, 2020

\begin{abstract}
Introduction: Although D-dimer was reported to be elevated in COVID-19 death, few studies about changes in serial DDimer Levels with different severity of illness. Early prophylactic anticoagulation contributes to improve patient outcomes in COVID-19, the perception of timing of anticoagulant treatment is mixed. Methods: Retrospective analysis of 38 severity COVID-19 patients, compared changes in serial D-Dimer Levels between low-flow oxygen therapy and higher concentration oxygen therapy. Patients were stratified into two groups distinguished by D-Dimer value in start of anticoagulation treatment, definition D-Dimer $=3 \mathrm{mg} / \mathrm{L}$ of a cut-off value. Compare the improvement within 7 and 14 days, the time to the first appearance of improvement with chest CT and the days of hospital stay between two groups. Results: A total of 38 severe patients who accept anticoagulant therapy were enrolled into the study from consecutive 59 confirmed cases. Regression analysis showed that compared to those D-Dimer[?]3mg/L, patients with higher D-Dimer increase the risk (OR=15.697, $\left.\mathrm{P}_{\mathfrak{0}} 0.001\right)$ to develop to further severe illness. The difference of patient's improvement within 14 days $(\mathrm{P}=0.043)$ was much more significant compared to 7 days $(\mathrm{P}=0.757)$ in two groups. The days at the time of $\mathrm{CT}$ imaging improvement were $12.1 \pm 3.6$ days in the D-Dimer[?]3, while 16.2+-6.4 days in D-Dimer 33 ( $\mathrm{p}=0.028)$. The hospital stays in the two groups were $27(25.5-30.5)$ days and 28 (27-36.5) days separately $(\mathrm{p}=0.086)$. Conclusions: D-dimer is available for the evaluation of illness condition and the timing of anticoagulant therapy in severe COVID-19, and its role should not be ignored and required further study.
\end{abstract}

\section{Introduction}

COVID-19 ${ }^{1}$ has spread around the world. With the efforts of various countries and international organizations, the epidemic has gradually controlled, but there are still no specific drugs for COVID-19. Improving symptoms and preventing further exacerbation of the disease has become critical to clinical treatment.

Most of studies have shown that D-Dimer levels in death with COVID-19 are increased significantly ${ }^{2}$, suggesting a possibility of the complication of venous thromboembolism (VTE). Autopsy found many microscopic lung thrombis ${ }^{3}$ and deep vein thrombosis (DVT) rates up to $25 \%$ in severe COVID-19 clinical findings ${ }^{4}$ indicating the risk of VTE is high in COVID-19. The COVID-19 is highly infectious and pathogenic which has increased health care workers' exposure risk in traditional approach for VTE diagnosis. In this special period, increasing the clinical awareness and diagnosis of VTE, focusing on the occurrence of early-stage clinical symptoms, using available laboratory test to evaluate the condition, and beginning anticoagulant therapy early ${ }^{5}$ while ensuring the safety is of great importance.

Current studies conducted that anticoagulant therapy can reduce COVID-19 mortality and improve patient prognosis ${ }^{6}$, but there are separate opinions about the timing of preventive anticoagulant therapy. Through the retrospective study, our team analyzed the dynamic changes of D-dimer under different illness conditions and the prognosis of anticoagulant therapy at different D-dimer levels. The aim of our study was to analyze the clinical significance of D-Dimer in predicting illness severity and guiding anticoagulant therapy in COVID-19.

\section{Methods}


This is a retrospective cohort study of 59 patients with confirmed COVID-19 pneumonia hospitalized at Infection Division of Union Hospital, Huazhong University of Science and Technology in Wuhan, China from January 25 to February 15, 2020. All patients were diagnosed with COVID-19 pneumonia according to World Health Organization interim guidance ${ }^{7}$. A retrospective review of the characteristics of these patients during the whole hospital period was performed through the electronic medical record system of our hospital, the last was monitored up to March 15, 2020. The ethics committee of Union Hospital (Wuhan, China) approved this study.

The clinical classification of COVID-19 reference the Diagnosis and Treatment Plan of COVID-19 suggested by National Health Commission of $\mathrm{China}^{8}$ (the seventh edition). The ordinary COVID-19 was defined to with fever, respiratory or other symptoms, but pneumonia asterisk in imaging procedure. The severe COVID19 was defined as meeting any one of following items: Respiratory rate[?]30 breaths /min; Arterial oxygen saturation[?] $93 \%$ at rest; $\mathrm{PaO} 2 / \mathrm{FiO} 2[?] 300 \mathrm{mmHg}$. All patients were treated with supportive care whatever they may needed respectively, including administration of oxygen (nasal cannula, noninvasive mechanical ventilation, invasive mechanical ventilation (IMV), or IMV with extra corporeal membrane oxygenation), antibiotic, antiviral and anticoagulant therapy, immunomodulator or methylprednisolone and so on. All of patients who were treated anticoagulant therapy in this study were precautionary, Low Molecular Weight Heparin (LMWH) (40-60mg enoxaparin/day) for 6 days at least or longer. Patients underwent blood routine blood test, coagulation, and biochemical tests and chest x-rays or computed tomography many times. For example, D-Dimer monitoring per 2-4d until it became stable and normal. It should be noted that all the clinical data used in this study were collected from the first day of hospital admission except D-Dimer that from the diverse stages of the diseases many times.

The evaluation of outcomes consisted of the time of CT imaging improvement was observed first time, hospital-stay time and the clinical improvement during 7 days and 14 days, defined as the change in oxygen support reference the seven-category ordinal scale ${ }^{9}$. The seven-category ordinal scale consisted of the following categories: 1, not hospitalized with resumption of normal activities; 2 , not hospitalized, but unable to resume normal activities; 3 , hospitalized, not requiring supplemental oxygen; 4, hospitalized, requiring supplemental oxygen; 5 , hospitalized, requiring nasal high flow oxygen therapy, noninvasive mechanical ventilation, or both; 6, hospitalized, requiring ECMO, invasive mechanical ventilation, or both; and 7, death.

Normally and abnormally distributed quantitative variables were compared using the Student's t-test and the Mann-Whitney U test, respectively. Categorical variables were compared using the chi-squared test. The results were given as the mean \pm standard deviation, median (interquartile range), or number (percentage), wherever appropriate. The level of the D-Dimer was reviewed by bivariate logistic regression analysis for their ability to predict states of illness. Survival curves were developed using the Kaplan-Meier method with log-rank test. A P valuej0.05 was considered statistically significant. All analyses were performed with SPSS 25.0 for windows.

\section{Results}

A total of 38 severe patients who accept anticoagulant therapy were enrolled into the study from consecutive 59 confirmed cases. Except 16 ordinary patients were not treated with anticoagulant therapy, there were 4 serve patients complicated with atrial fibrillation and 1 with acute myocardial infraction were also excluded to the study even they were treated with anticoagulant therapy (Figure 1).

$22(57.9 \%)$ severe patients recovered with low-flow nasal cannula (LFNC) only. 16(42.1\%) severe patients' condition worsened, they need higher oxygen support therapy, of which eleven with high flow nasal cannula (HFNC) or noninvasive mechanical ventilation and five with invasive mechanical ventilation were consisted. Continuous D-Dimer monitoring showed a significant difference $(\mathrm{P} ; 0.001)$ between the stage of LFNC (2.01(1.37-2.89)mg/L) and HFNC (13.57(7.33-19.84)mg/L) (Table.1). Using binary logistic regression analysis evaluate the influence of D-Dimer level on degree of patient's condition found that compared to those D-Dimer[?]3mg/L, patients with higher D-Dimer increase the risk ( $\left.\mathrm{OR}=15.697, \mathrm{P}_{j} 0.001\right)$ to develop to much more severe illness (Table.2). 
The mean age at disease onset of the total $(\mathrm{n}=38)$ was $69.4+-12.2$ years, incidence time was 10.6+-6.2 days, and $21(55.3 \%)$ were male. $21(55.3 \%)$ patients had one or more comorbidities, including hypertension, diabetes and heart disease. All patients were treated with preventative anticoagulant therapy, eighteen when D-Dimer[?]3mg/L and twenty until D-Dimer $33 \mathrm{mg} / \mathrm{L}$. The parameters of clinical characteristics and laboratory tests between two parts were compared (Table.3).

According to the change in oxygen support reference the seven-category ordinal scale, 10 (55.6\%) patients' condition improvement in 7 days in the group of D-Dimer [?]3 when anticoagulant therapy was initiated, while $7(35 \%)$ in the D-Dimer i3 $(\mathrm{P}=0.757) .17(83 \%)$ patients' condition improvement in 14 days in the D-Dimer[?]3 compared with $10(50 \%)$ in the D-Dimer ¿3 (P=0.043, Table.4). Then time to clinical improvement were examined, there was a difference in 14 days of improvement between D-Dimer[?]3 and D-Dimer ¿3 $(\mathrm{P}=0.03)$, while no difference in 7 days $(\mathrm{P}=0.24$, Figure.2).

The mean days at the time of CT imaging improvement was 12.1+-3.6 days in the group of D-Dimer[?]3, while 16.2+-6.4 days in D-Dimer ¿3 $(\mathrm{p}=0.028)$. The hospital stays in the two groups were 27 (25.5-30.5) days and 28 (27-36.5) days separately, there was no statistically significant $(\mathrm{p}=0.086$, Figure. 3$)$.

\section{Conclusion}

D-dimer is available for the evaluation of illness condition and the timing of anticoagulant therapy in severe COVID-19, and its role should not be ignored and required further study.

\section{Discussion}

D-dimer is a biomarker of fibrin degradation that can be measured in plasma. D-Dimer content of normal human plasma is very low, but is increased relating to thrombosis. Plasma D-Dimer level has high sensitivity in the diagnosis of VTE, but with modest specificity. It can be confused by numerous pathophysiological events, such as inflammation, malignancy, pregnancy, myocardial infarction, DIC, aortic dissection, and liver and kidney dysfunction ${ }^{10}$.

In our study, we report the levels of D-Dimer spread around $1.37-2.89 \mathrm{mg} / \mathrm{L}$ in the stage of low-flow oxygen in COVID-19, may be caused by inflammation, and (or) thrombosis, or other factors. An obvious increasing trend can be found at the D-Dimer levels while the condition of the patient deteriorated further. Moreover, risk of aggravation of COVID-19 with D-Dimer $33 \mathrm{mg} / \mathrm{L}$ is more than 15 times than those D-Dimer[?]3mg/L. Though lack multi-factor regression analysis, other researchers have reported similar associations. Guan and Tang reported the significant increase with D-Dimer in COVID-19 death ${ }^{11}$. Wu found D-Dimer was a risk factors associated with the development of acute respiratory distress syndrome (ARDS) and progression from ARDS to death ${ }^{12}$. Despite the reason is various and complicated for D-Dimer abnormalities in COVID-19, disease aggravation accompanied by increased plasma D-Dimer levels. It is conceivable that D-Dimer may be an effective parameter to assess the degree of illness.

Whether the D-Dimer abnormalities were directly responsible for VTE cannot be determined, as its modest specificity. The high infectivity and pathogenicity of SARS-CoV-2 limit the routine screening of VTE by Doppler studies of lower limb veins and pulmonary artery CTA. Diagnosis should be decided according to the peculiar circumstances and available related tests of the patients, and how to consider D-Dimer abnormalities in perspective may provide new ideas for identifying VTE. Inflammatory reactions can activate the coagulation system, but some differences were found in Zhou's research that elevated D-dimer levels occurred earlier than that of inflammatory factor, suggesting that the very high D-dimer levels observed in COVID-19 patients are not only secondary to systemic inflammation, but also reflect true thrombotic disease, possibly induced by cellular activation that is triggered by the virus ${ }^{13-14}$. In addition, a study reported that the incidence of VTE in severe COVID-19 patients in the Intensive Care Unit (ICU) was 25\% ${ }^{4}$. Besides, $30 \%$ patients had acute pulmonary embolus when pulmonary CT angiograms performed for COVID-19 patients showed in a study of France with 106 patients $^{15}$. In a cohort study of Wang forecasted that $40 \%$ of COVID19 patients had a high risk of $\mathrm{VTE}^{16}$. Unlike SARS-CoV, small vessel thrombosis was found not only in pulmonary but also in multiple organs after SARS-CoV-2 infection in humans ${ }^{13}$. Based on a comprehensive 
analysis of these consequences, it was hypothesized that D-Dimer abnormalities and VTE in COVID-19 had a close relationship. It should be noted that, in one interesting study, plasmin may cleave a newly inserted furin site in the S protein of SARS-CoV-2, extracellularly, which increases its infectivity and virulence ${ }^{17}$. Elevated plasmin is a shared feature in people with underlying medical conditions, including hypertension, diabetes, cardiovascular disease, cerebrovascular disease, and chronic renal illness. Those people are susceptible to SARS-CoV-2 infection, their elevated plasmin was responsible for D-Dimer abnormalities.

In addition to its assessment of risk and diagnostic use, D-Dimer has been proposed to guide anticoagulant therapy gradually. Tang et al ${ }^{18}$ showed that the prophylactic anticoagulation regimen can reduce the COVID19 patient mortality when D-Dimer levels greater than six times of the normal upper limit value. What is more, Lin et al ${ }^{19}$ suggested beginning prophylactic anticoagulation regimen once D-Dimer up to four times of the normal. Larger prospective studies are to be required to support the D-Dimer value can become guidelines on anticoagulant therapy in the future, but now its severity of the abnormal value and rate of the significant change can provide a basis and direction for clinical treatment. In our present study, in patients on anticoagulant therapy, the recovery was quick of those plasma D-Dimer[?]3mg/L when anticoagulant therapy was initiated than that of plasma D-Dimer $33 \mathrm{mg} / \mathrm{L}$. Nevertheless, their hospital stays had no significant difference, it may be related to our discharge criteria that the condition was significantly alleviated and nucleic acid tests were negative. Viral infection is a self-limiting disease, the anticoagulant role of LMWH may alleviate the illness but does not have a prominent antiviral function.

However, due to the limited number of cases included in our study, especially hard to collect the matched no anticoagulant therapy patients, we could not perform further and more conceivable analysis. Through the analysis of D-Dimer changes at different stages of illness and its function to suggest much more benefits could be made at early-stage anticoagulation therapy in severe COVID-19, the guidance of D-Dimer should not be ignored and should be studied further.

\section{Funding}

We would like to thank the National Natural Science Foundation of China. (No. 81900432)

\section{Declaration of competing interest}

The authors have no proprietary or commercial conflicts of interest to disclose.

\section{References:}

1. Chen A, Coura-Filho GB, Rehder M. Clinical Characteristics of Covid-19 in China. N Engl J Med 2020;382:1860.

2. Terpos E, Ntanasis-Stathopoulos I, Elalamy I, et al. Hematological findings and complications of COVID19. American Journal of Hematology 2020.

3. Zhang T, Sun LX, Feng RE. [Comparison of clinical and pathological features between severe acute respiratory syndrome and coronavirus disease 2019]. Zhonghua Jie He He Hu Xi Za Zhi 2020;43:E040.

4. Cui S, Chen S, Li X, et al. Prevalence of venous thromboembolism in patients with severe novel coronavirus pneumonia. Journal of Thrombosis and Haemostasis 2020.

5. Li T, Lu H, Zhang W. Clinical observation and management of COVID-19 patients. Emerg Microbes Infect 2020;9:687-690.

6. Kollias A, Kyriakoulis KG, Dimakakos E, et al. Thromboembolic risk and anticoagulant therapy in COVID-19 patients: emerging evidence and call for action. Br J Haematol 2020.

7. World Health Organization. Clinical management of severe acute respiratory infection when novel coronavirus (nCoV) infection is suspected: interim guidance. Published January 28, 2020. Accessed January 31, 2020. https://www.who.int/publications-detai 
8. National Health Commission of China. The diagnosis and treatment plan for the novel coronavirus disease (the seventh edition). 2020.

9. Coronavirus disease (COVID-2019) R\&D. Geneva: World Health Organization. https://www.who.int/blueprint/ priority-diseases/key -action/novel -coronavirus/en/

10. Weitz JI, Fredenburgh JC, Eikelboom JW. A Test in Context: D-Dimer. Journal of the American College of Cardiology 2017;70:2411-2420.

11. Tang N, Li D, Wang X, Sun Z. Abnormal coagulation parameters are associated with poor prognosis in patients with novel coronavirus pneumonia. Journal of Thrombosis and Haemostasis 2020;18:844-847.

12. Wu C, Chen X, Cai Y, et al. Risk Factors Associated With Acute Respiratory Distress Syndrome and Death in Patients With Coronavirus Disease 2019 Pneumonia in Wuhan, China. JAMA Intern Med 2020.

13. Oudkerk M, Buller HR, Kuijpers D, et al. Diagnosis, Prevention, and Treatment of Thromboembolic Complications in COVID-19: Report of the National Institute for Public Health of the Netherlands. Radiology 2020:201629.

14. Z Varga: Endothelial cell infection and endotheliitis in COVID-19. The Lancet's website. https://www.thelancet.com/lancet/article/s0140-6736(20)30937-5. Published April 17, 2020. Assessed April 19,2020 .

15. LEONARD-LORANT L, Xavier D, HELMS, et al. Acute Pulmonary Embolism in COVID-19 Patients on CT Angiography and Relationship to D-Dimer Levels. Radiology 2020.

16. Wang T, Chen R, Liu C, et al. Attention should be paid to venous thromboembolism prophylaxis in the management of COVID-19. The Lancet Haematology 2020;7:e362-e363.

17. Ji H, Zhao R, Matalon S, et al. Elevated Plasmin(ogen) as a Common Risk Factor for COVID-19 Susceptibility. Physiological Reviews 2020;100:1065-1075.

18. Tang N, Bai H, Chen X, et al. Anticoagulant treatment is associated with decreased mortality in severe coronavirus disease 2019 patients with coagulopathy. J Thromb Haemost 2020;18:1094-1099.

19. Lin L, Lu L, Cao W, et al. Hypothesis for potential pathogenesis of SARS-CoV-2 infection-a review of immune changes in patients with viral pneumonia. Emerg Microbes Infect 2020;9:727-732.

\section{Figure legends}

Figure.1 Study profile

Figure. 2 Time to Clinical Improvement analysis in different D-Dimer levels when anticoagulant therapy was initiated. The differences of improvement in two groups were observed both within 7 days $(\mathrm{P}=0.24)$ and 14 days $(\mathrm{P}=0.03)$.

Figure. 3 The time to the first appearance of improvement with chest CT and patients' hospital stays between two groups. The mean days at the time of CT imaging improvement were 12.1+-3.6 days in the group of D-Dimer[?]3, while 16.2+-6.4 days in D-Dimer $33(\mathrm{p}=0.028)$. The hospital stays in the two groups were $27(25.5-30.5)$ days and $28(27-36.5)$ days separately $(\mathrm{p}=0.086)$.

\section{Hosted file}

Tables.docx available at https://authorea.com/users/347100/articles/472916-attention-shouldbe-paid-to-d-dimer-test-in-the-management-of-covid-19 

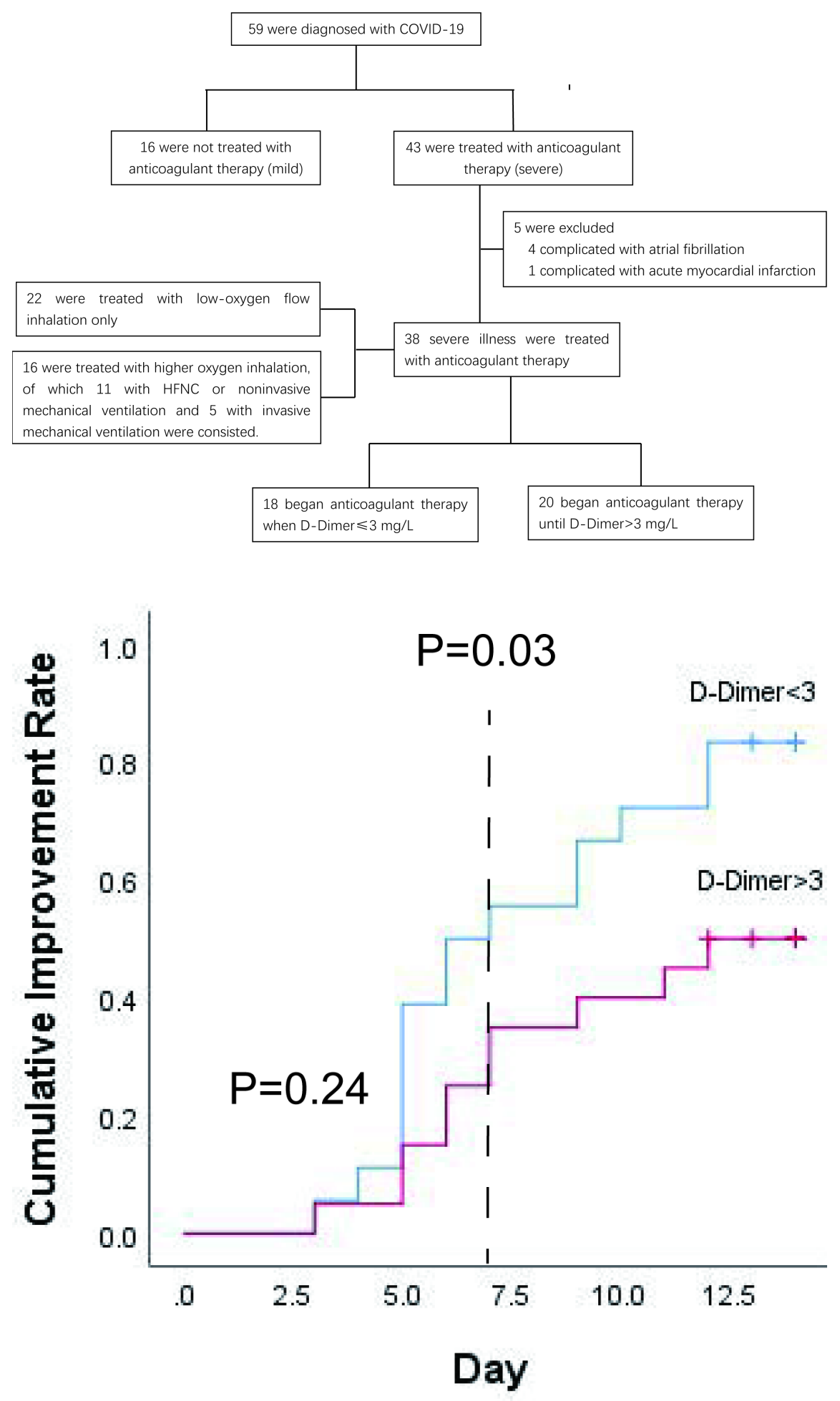
A

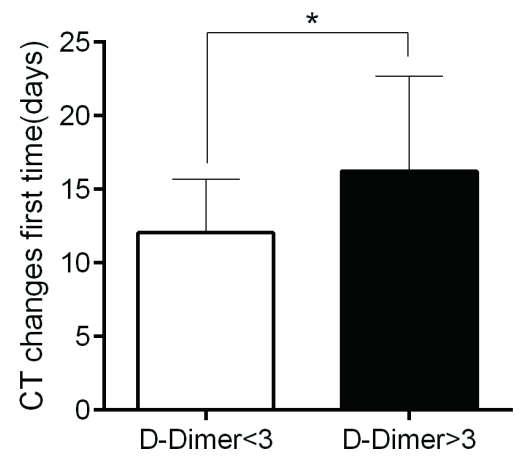

B

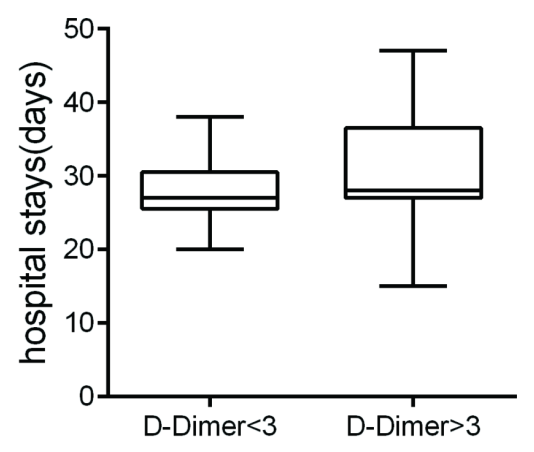

